Nouvelles perspectives en sciences sociales

Revue internationale de systémique complexe et d'études relationnelles

\title{
De l'interdisciplinarité à la transdisciplinarité : fondation méthodologique du dialogue entre les sciences humaines et les sciences exactes
}

\section{Basarab Nicolescu}

Volume 7, numéro 1, octobre 2011

Sur le thème de l'interdisciplinarité

URI : https://id.erudit.org/iderudit/1007083ar

DOI : https://doi.org/10.7202/1007083ar

Aller au sommaire du numéro

Éditeur(s)

Prise de parole

ISSN

1712-8307 (imprimé)

1918-7475 (numérique)

Découvrir la revue

Citer cet article

Nicolescu, B. (2011). De l'interdisciplinarité à la transdisciplinarité : fondation méthodologique du dialogue entre les sciences humaines et les sciences exactes. Nouvelles perspectives en sciences sociales, 7(1), 89-103.

https://doi.org/10.7202/1007083ar
Résumé de l'article

Le texte analyse la manière dont la transdisciplinarité peut conduire à une fondation méthodologique du dialogue entre deux cultures post-modernes (culture technoscientifique et culture spirituelle) de même qu'entre les sciences humaines et les sciences exactes. 


\title{
De l'interdisciplinarité à la transdisciplinarité : fondation méthodologique du dialogue entre les sciences humaines et les sciences exactes
}

\author{
Basarab Nicolescu \\ Centre international de recherches et \\ études transdisciplinaires, Paris \\ et Université Babes-Bolyai, Cluj-Napoca
}

\section{Deux cultures post-modernes}

u début de l'histoire humaine, science et culture étaient A inséparables. Les mêmes interrogations sur le sens de l'Univers et de la vie les animaient.

À la Renaissance, le lien n'était pas encore rompu. La première Université, comme son nom l'indique, était censée étudier l'universel. L'universel était incarné en ceux qui marquèrent du sceau de leur œuvre l'histoire de la connaissance. La rupture entre la science et le sens, entre le Sujet et l'Objet, était présente, certes, en germe au XVII e siècle, quand la méthodologie de la science moderne fut formulée, mais elle n'est devenue visible qu'au XIX ${ }^{e}$ siècle, quand le big-bang disciplinaire prit son essor.

De nos jours, la rupture est consommée. Science et culture n'ont plus rien en commun; c'est pourquoi d'ailleurs on parle de la science et de la culture, des sciences exactes ou dures (comme 
si les sciences humaines étaient inexactes) et des sciences humaines ou molles (comme si les sciences exactes étaient inhumaines ou surhumaines). La science n'a pas accès à la noblesse de la culture et la culture n'a pas accès au prestige de la science.

On comprend le tollé déclenché par le concept de deux cultures - la culture scientifique et la culture humaniste - introduit il y a quatre décennies par Charles Percy Snow, à la fois romancier et homme de science ${ }^{1}$. La science est bien une partie de la culture, mais cette culture scientifique est complètement séparée de la culture humaniste. Les deux cultures sont perçues comme antagonistes. Chaque monde - le monde scientifique ou le monde humaniste - est hermétiquement clos sur lui-même.

Ces derniers temps, les signes de rapprochement entre les deux cultures, entre sciences humaines et sciences exactes, se multiplient, surtout dans le domaine du dialogue entre la science et l'art, axe fondateur du dialogue entre la culture scientifique et la culture humaniste. Mais cette réconciliation est-elle possible?

La situation est aujourd'hui encore plus compliquée qu'en 1959, quand Snow a formulé le concept de deux cultures. Le mariage entre la science fondamentale et la technologie est maintenant consommé, en engendrant la culture technoscientifique. Cette nouvelle culture est la source de la force irrationnelle de la mondialisation sans visage humain, centrée sur l'économie et qui peut effacer toute différence entre les cultures et entre les religions. La culture humaniste a été déjà, en grande partie, phagocytée par la culture technoscientifique. Devant cette nouvelle culture monolithique se dresse ce que j'appelle la culture spirituelle, qui est, en fait, une constellation d'une grande hétérogénéité de cultures, de religions et de communautés spirituelles. La culture spirituelle, malgré sa nature contradictoire, partage une foi commune dans la double nature de l'être humain : d'une part, sa nature physique, biologique et psychique et, d'autre part, sa nature transcendante.

Charles Percy Snow, The Two Cultures, Cambridge University Press, Cambridge, 1993. Ce livre est fondé sur une conférence donnée par Charles Percy Snow en 1959. 
Comme scientifiques et penseurs, participants actifs à la culture technoscientifique, nous avons une grande responsabilité : bâtir d'urgence les ponts entre la culture technoscientifique et la culture spirituelle. Mais, ces ponts sont-ils concevables ou ne s'agit-il que d'une illusion utopique?

Dans le présent texte, nous analysons comment la transdisciplinarité nous conduit à une fondation méthodologique du dialogue entre les deux cultures post-modernes et entre les sciences humaines et les sciences exactes.

\section{L’obstacle épistémologique sur la voie du dialogue : un seul ou plusieurs niveaux de Réalité?}

Devant le succès indiscutable de la science moderne, les sciences humaines ont forcément tenté sans cesse d'imiter les sciences exactes. La neutralité et l'objectivité sont devenues ainsi les deux critères du caractère scientifique de ce qu'on appelle les sciences humaines. L'objectif asymptotique, avoué ou inavoué, est la formalisation mathématique. En fait, ce qui est imité est la physique classique, aujourd'hui dépassée dans son horizon épistémologique. Il convient donc d'interroger ce qu'on entend par "science moderne".

La science moderne est née d'une rupture brutale avec l'ancienne vision du monde ${ }^{2}$. Elle est fondée sur l'idée, surprenante et révolutionnaire pour l'époque, d'une séparation totale entre le sujet-connaisseur et la Réalité, supposée être complètement indépendante du sujet qui l'observe. Mais, en même temps, la science moderne se donnait trois postulats fondamentaux, qui prolongeaient, à un degré suprême, sur le plan de la raison, la quête de lois et de l'ordre :

1. L'existence de lois universelles, de caractère mathématique.

2. La découverte de ces lois par l'expérience scientifique.

3. La reproductibilité parfaite des données expérimentales.

Un langage artificiel, différent du langage de la tribu - les mathématiques - était ainsi élevé, par Galilée, au rang de langage commun entre Dieu et les hommes.

2 Basarab Nicolescu, Nous, la particule et le monde, Rocher, Monaco, 2002 
Les succès extraordinaires de la physique classique, de Galilée, Kepler et Newton jusqu'à Einstein, ont confirmé la justesse de ces trois postulats. En même temps, ils ont contribué à l'instauration d'un paradigme de la simplicité, qui est devenu prédominant au seuil du XIX ${ }^{\mathrm{e}}$ siècle. La physique classique est parvenue à bâtir, au cours de deux siècles, une vision du monde apaisante et optimiste prête à accueillir, sur le plan individuel et social, le surgissement de l'idée de progrès.

La physique classique est fondée sur l'idée de continuité, en accord avec l'évidence fournie par les organes des sens : on ne peut pas passer d'un point à l'autre de l'espace et du temps sans passer par tous les points intermédiaires.

L'idée de continuité est intimement liée à un concept-clé de la physique classique : la causalité locale. Tout phénomène physique pouvait être compris par un enchaînement continu de causes et d'effets : à chaque cause à un point donné correspond un effet à un point infiniment proche et à chaque effet à un point donné correspond une cause à un point infiniment proche. On n'a point besoin d'une quelconque action directe à distance.

Le concept de déterminisme pouvait faire ainsi son entrée triomphale dans l'histoire des idées. Les équations de la physique classique sont telles que, si on connaît les positions et les vitesses des objets physiques à un moment donné, on peut prédire leurs positions et leurs vitesses à n'importe quel autre moment du temps. Les lois de la physique classique sont des lois déterministes. Les états physiques étant des fonctions de positions et de vitesses, il en résulte que si l'on précise les conditions initiales (l'état physique à un moment donné du temps) on peut prédire complètement l'état physique à n'importe quel autre moment donné du temps.

Il est bien évident que la simplicité et la beauté esthétique de tels concepts - continuité, causalité locale, déterminisme - si opératifs dans la Nature, aient fasciné les plus grands esprits.

Il restait un pas à franchir qui n'était plus de nature scientifique mais de nature philosophique et idéologique : proclamer la physique reine des sciences. Plus précisément, tout réduire à la 
physique, le biologique et le psychique n'apparaissant que comme des étapes évolutives d'un seul et même fondement. Ce pas a été facilité par les avancées indiscutables de la physique. Ainsi est née l'idéologie scientiste qui est apparue comme une idéologie d'avant-garde et qui a connu un extraordinaire essor au XIX siècle.

Sur le plan académique, les conséquences du scientisme ont été aussi considérables. Une connaissance digne de ce nom ne peut être que scientifique, objective. Toute connaissance autre que scientifique est repoussée dans l'enfer de la subjectivité.

L'objectivité, érigée en critère suprême de vérité, a eu une conséquence inévitable : la transformation du sujet en objet. La mort de l'homme, qui annonce tant d'autres morts, est le prix à payer pour une connaissance dite " objective ». Au fond, au delà de l'immense espoir qu'il a soulevé, le scientisme nous a légué une idée persistante et tenace : celle de l'existence d'un seul niveau de Réalité.

Une nouvelle vision du monde - la vision quantique - allait ruiner les fondements d'une pensée qui n'en finit pas de finir.

Au seuil du $\mathrm{XX}^{\mathrm{e}}$ siècle, Max Planck, fut confronté à un problème de physique, d'apparence innocente, comme tous les problèmes de physique. Mais, pour le résoudre, il fut conduit à une découverte qui provoqua en lui, selon son propre témoignage, un véritable drame intérieur. Car il était devenu le témoin de l'entrée de la discontinuité dans le domaine de la physique. Selon la découverte de Planck, l'énergie a une structure discrète, discontinue. Le quantum de Planck, qui a donné son nom à la mécanique quantique, allait révolutionner toute la physique et changer en profondeur notre vision du monde.

Comment comprendre la vraie discontinuité, c'est-à-dire imaginer qu'entre deux points il n'y a rien, ni objets, ni atomes, ni molécules, ni particules, juste rien? Et même le mot "rien" est de trop.

Mettre en question la continuité revenait à mettre en question la causalité locale et à ouvrir ainsi une redoutable boîte de Pandore. 
Une quantité physique a, selon la mécanique quantique, plusieurs valeurs possibles, affectées de probabilités bien déterminées. Mais dans une mesure expérimentale on obtient, bien évidemment, un seul résultat pour la quantité physique en question. Cette abolition brusque de la pluralité des valeurs possibles d'une " observable " physique, par l'acte de mesure, avait une nature obscure, mais elle indiquait clairement l'existence d'un nouveau type de causalité.

Sept décennies après la naissance de la mécanique quantique, la nature de ce nouveau type de causalité a été éclaircie grâce à un résultat théorique rigoureux - le théorème de Bell - et à des expériences d'une grande précision. Un nouveau concept faisait ainsi son entrée dans la physique : la non-séparabilité.

Les entités quantiques continuent d'interagir quel que soit leur éloignement. Un nouveau type de causalité apparaît ainsi dans l'histoire de la connaissance - une causalité globale qui concerne le système de toutes les entités physiques, dans leur ensemble. Un autre pilier de la pensée classique - le déterminisme - allait, à son tour, s'écrouler.

Les entités quantiques - les quantons - sont à la fois corpuscules et ondes ou, plus précisément, ils ne sont ni particules ni ondes. Les célèbres relations de Heisenberg montrent, sans aucune ambiguïté, qu'il est impossible de localiser un quanton dans un point précis de l'espace et dans un point précis du temps. Autrement dit, il est impossible d'assigner une trajectoire bien déterminée à une particule quantique. L'indéterminisme régnant à l'échelle quantique est un indéterminisme constitutif, fondamental, irréductible qui ne signifie nullement hasard ou imprécision. L'impact majeur culturel de la révolution quantique est certainement la remise en cause du dogme philosophique contemporain de l'existence d'un seul niveau de Réalité ${ }^{3}$.

Donnons au mot " réalité " son sens à la fois pragmatique et ontologique. Nous entendons par Réalité, tout d'abord, ce qui résiste à nos expériences, représentations, descriptions, images ou formalisations mathématiques. La physique quantique nous a fait

3 Basarab Nicolescu, Qu'est-ce que la Réalité?, Liber, Montréal, 2009 
découvrir que l'abstraction n'est pas un simple intermédiaire entre nous et la Nature, un outil pour décrire la réalité, mais une des parties constitutives de la Nature.

Il faut donner une dimension ontologique à la notion de Réalité, dans la mesure où la Nature participe de l'être du monde. La Réalité n'est pas seulement une construction sociale, le consensus d'une collectivité, un accord intersubjectif. Elle a aussi une dimension trans-subjective, dans la mesure ou un simple fait expérimental peut ruiner la plus belle théorie scientifique.

Il faut entendre par niveau de Réalité un ensemble de systèmes invariant à l'action d'un nombre de lois générales : par exemple, les entités quantiques soumises aux lois quantiques, lesquelles sont en rupture radicale avec les lois du monde macrophysique. C'est dire que deux niveaux de Réalité sont différents si, en passant de l'un à l'autre, il y a rupture des lois et rupture des concepts fondamentaux (comme, par exemple, la causalité). Personne n'a réussi à trouver un formalisme mathématique qui permet le passage rigoureux d'un monde à l'autre.

En fait, Werner Heisenberg, dans ces écrits philosophiques, s'est beaucoup rapproché du concept de "niveau de Réalité ". Dans son célèbre Manuscrit de 1942 (publié en allemand seulement en 1984 et traduit en français en 1998), Heisenberg, qui a bien connu Husserl, introduit l'idée de trois régions de la réalité, aptes à nous fournir l'accès au concept de "réalité " lui-même : la première région est celle de la physique classique, la deuxième, de la physique quantique et des phénomènes biologiques et psychiques et, la troisième, celle des expériences religieuses, philosophiques et artistiques ${ }^{4}$. Cette classification a un fondement subtil : celui de la proximité de plus en plus grande entre le Sujet et l'Objet.

L'émergence de niveaux de Réalité différents dans l'étude des systèmes naturels est un événement capital dans l'histoire de la connaissance. Elle peut nous conduire à repenser le dialogue

Werner Heisenberg, Philosophie - Le manuscrit de 1942, trad. de l'allemand et introduction par Catherine Chevalley, Paris, Seuil, 1998 [éd. allemande :1984], p. 218-306. 
entre les sciences humaines et les sciences exactes. La transdisciplinarité m’apparaît comme le médiateur inévitable de ce dialogue.

\section{Multi-, inter- et transdisciplinarité}

La croissance sans précédent des savoirs à notre époque rend légitime la question de l'adaptation des mentalités à ces savoirs. Tout particulièrement, la mondialisation est une source potentielle d'une nouvelle décadence. Les deux dangers extrêmes de la mondialisation sont l'homogénéisation culturelle, religieuse et spirituelle et le paroxysme des conflits ethniques et religieux, comme réaction d'autodéfense des cultures et des civilisations.

L'harmonie entre les mentalités et les savoirs présuppose que ces savoirs soient intelligibles, compréhensibles. Mais une compréhension peut-elle encore exister à l'ère du big-bang disciplinaire et de la spécialisation à outrance?

Le besoin indispensable de liens entre les différentes disciplines s'est traduit par l'émergence, vers le milieu du XX $\mathrm{X}^{\mathrm{e}}$ siècle, de la pluridisciplinarité et de l'interdisciplinarité.

La pluridisciplinarité concerne l'étude d'un objet d'une seule et même discipline par plusieurs disciplines à la fois. La recherche pluridisciplinaire apporte un plus à la discipline en question, mais ce "plus " est au service exclusif de cette même discipline.

L'interdisciplinarité concerne le transfert des méthodes d'une discipline à l'autre. Par exemple, le transfert des méthodes de la mathématique aux phénomènes météorologiques ou ceux de la bourse a engendré la théorie du chaos. L'interdisciplinarité déborde les disciplines, mais sa finalité reste aussi inscrite dans la recherche disciplinaire.

La transdisciplinarité concerne, comme le préfixe latin trans l'indique, ce qui est à la fois entre les disciplines, à travers les différentes disciplines et au delà de toute discipline. Sa finalité est la compréhension du monde présent, dont un des impératifs est l'unité de la connaissance. Le mot « transdisciplinarité » a été introduit en 1970 par Jean Piaget.

5 Jean Piaget, "L'épistémologie des relations interdisciplinaires " dans 
La recherche transdisciplinaire est radicalement distincte de la recherche disciplinaire, tout en lui étant complémentaire. La recherche disciplinaire concerne, tout au plus, un seul et même niveau de Réalité; d'ailleurs, dans la plupart des cas, elle ne concerne que des fragments d'un seul et même niveau de Réalité. En revanche, la transdisciplinarité s'intéresse à la dynamique engendrée par l'action de plusieurs niveaux de Réalité à la fois. La découverte de cette dynamique passe nécessairement par la connaissance disciplinaire.

La disciplinarité, la pluridisciplinarité, l'interdisciplinarité et la transdisciplinarité sont les quatre flèches d'un seul et même arc : celui de la connaissance.

La connaissance plénière est un nouveau type de connaissance - la connaissance transdisciplinaire (CT), qui correspond à une connaissance in vivo. Cette nouvelle connaissance est concernée par la correspondance entre le monde extérieur de l'Objet et le monde intérieur du Sujet. La connaissance CT est réellement une connaissance du tiers. Par définition, la connaissance CT inclut un système de valeurs (voir Tableau 1).

Il est important de réaliser que la connaissance disciplinaire et la connaissance transdisciplinairene ne sont pas antagonistes mais complémentaires. Leurs deux méthodologies sont fondées sur l'esprit scientifique.

La transdisciplinarité est une voie de témoignage de notre présence au monde et de notre expérience vécue à travers les fabuleux savoirs de notre époque. La vision transdisciplinaire, qui est à la fois une vision transculturelle et transreligieuse, conduit, sur le plan social, à un changement radical de perspective et d'attitude.

L'interdisciplinarité; Problèmes d'enseignement et de recherche dans les universités, OCDE, Paris, 1972, p. 131-144. 


\section{Tableau 1}

Comparaison entre les connaissances disciplinaire (CD) et transdisciplinaire (CT)

\begin{tabular}{|c|c|}
\hline $\begin{array}{c}\text { CONNAISSANCE } \\
\text { DISCIPLINAIRE (CD) }\end{array}$ & $\begin{array}{c}\text { CONNAISSANCE } \\
\text { TRANSDISCIPLINAIRE (CT) }\end{array}$ \\
\hline IN VITRO & IN VIVO \\
\hline monde externe - Objet & $\begin{array}{c}\text { correspondance entre le monde externe } \\
\text { (Objet) } \\
\text { et le monde interne (Sujet) }\end{array}$ \\
\hline savoir & compréhension \\
\hline intelligence analytique & $\begin{array}{c}\text { nouveau type d'intelligence - équilibre entre } \\
\text { le mental, les sentiments et le corps }\end{array}$ \\
\hline orienté vers le pouvoir et la possession & orienté vers l'étonnement et le partage \\
\hline logique binaire & logique du tiers inclus \\
\hline exclusion des valeurs (neutralité) & inclusion des valeurs (option humaniste) \\
\hline
\end{tabular}

\section{Méthodologie de la transdisciplinarité}

La méthodologie de la transdisciplinarité ${ }^{6}$ est fondée sur trois axiomes :

i. L'axiome ontologique : L'existence de différents niveaux de Réalité de l'Objet et de différents niveaux de Réalité du Sujet.

ii. L'axiome logique : Le passage d'un niveau de Réalité à un autre niveau de Réalité s'effectue par la logique du tiers inclus.

iii. L'axiome épistémologique : La structure de l'ensemble des niveaux de Réalité apparait, dans notre connaissance de la nature, de la société et de nous-mêmes, comme une structure complexe : chaque niveau est ce qu'il est parce que tous les autres niveaux existent à la fois. 
J'ai analysé en détail la justification de ces axiomes dans le livre Qu'est-ce que la Réalité. Il convient de dire ici quelques mots sur la signification du deuxième axiome.

Deux niveaux différents de Réalité sont reliés par la logique $\mathrm{du}$ tiers inclus, une nouvelle logique par rapport à la logique classique.

La logique classique est fondée sur trois axiomes :

1. L'axiome d'identité : A est A.

2. L'axiome de non-contradiction : A n'est pas non-A.

3. L'axiome du tiers exclu : il n'existe pas un troisième terme $\mathrm{T}$ (T de "tiers inclus") qui est à la fois A et non-A.

$\mathrm{Si}$ on accepte néanmoins cette logique qui, après tout, a régné pendant deux millénaires et qui continue de dominer la pensée d'aujourd'hui, on arrive immédiatement à la conclusion que les couples de contradictoires mis en évidence par la physique quantique - onde et corpuscule, continuité et discontinuité, séparabilité et non-séparabilité, causalité locale et causalité globale, symétrie et brisure de symétrie, réversibilité et irréversibilité du temps, etc. - sont mutuellement exclusifs, car on ne peut affirmer en même temps la validité d'une chose et son contraire : A et non-A.

La plupart des logiques quantiques ont modifié le deuxième axiome de la logique classique - l'axiome de non-contradiction - en introduisant la non-contradiction à plusieurs valeurs de vérité à la place de celle du couple binaire (A, non-A). Ce fut le mérite historique de Stéphane Lupasco (1900-1988) d'avoir montré que la logique du tiers inclus est une véritable logique, formalisable et formalisée, multivalente (à trois valeurs : A, non-A et $\mathrm{T}$ ) et non-contradictoire ${ }^{7}$.

La compréhension de l'axiome du tiers inclus - il existe un troisième terme $\mathrm{T}$ qui est à la fois $\mathrm{A}$ et non- $\mathrm{A}$ - s'éclaire complètement lorsque la notion de "niveaux de Réalité " est introduite.

Stéphane Lupasco, Le principe d'antagonisme et la logique de l'énergie Prolégomènes à une science de la contradiction, Monaco, Le Rocher, coll. "L'esprit et la matière ", [1951] 1987. 
Pour obtenir une image claire du sens du tiers inclus, imaginons les trois termes de la nouvelle logique - A, non-A et $\mathrm{T}$ - et leurs dynamismes associés comme étant représentés par un triangle dont l'un des sommets se situe à un niveau de Réalité et les deux autres sommets à un autre niveau de Réalité. Si l'on reste à un seul niveau de Réalité, toute manifestation apparaît comme une lutte entre deux éléments contradictoires. Le troisième dynamisme, celui de l'état T, s'exerce à un autre niveau de Réalité, où ce qui apparaît comme désuni est en fait uni, et ce qui apparaît contradictoire est perçu comme non contradictoire. C'est la projection de $\mathrm{T}$ sur un seul et même niveau de Réalité qui produit l'apparence des couples antagonistes, mutuellement exclusifs.

La logique du tiers inclus est capable de décrire la cohérence entre tous les niveaux de Réalité par un processus itératif. Ce processus continuera à l'infini, jusqu'à l'épuisement de tous les niveaux de Réalité, connus ou concevables, sans jamais pouvoir aboutir à une théorie complètement unifiée. Dans ce sens, nous pouvons parler d'une évolution de la connaissance, sans jamais pouvoir aboutir à une non-contradiction absolue, impliquant tous les niveaux de Réalité : la connaissance est à jamais ouverte.

Il y a certainement une cohérence entre les différents niveaux de Réalité, tout du moins dans le monde naturel. En fait, une vaste autoconsistance - un bootstrap cosmique - semble régir l'évolution de l'univers, de l'infiniment petit à l'infiniment grand, de l'infiniment bref à l'infiniment long. Un flux d'information se transmet d'une manière cohérente d'un niveau de Réalité à un autre niveau de Réalité de notre univers physique. Cette cohérence est orientée : il y a une flèche associée à la transmission de l'information d'un niveau à l'autre. Par conséquent, la cohérence, si elle est limitée aux seuls niveaux de Réalité, s'arrête au niveau le plus « haut » et au niveau le plus «bas ». Pour que la cohérence continue au delà de ces deux niveaux limites, pour qu'il y ait une unité ouverte, il faut considérer que l'ensemble des niveaux de Réalité se prolonge par une zone de non-résistance à nos expé- 
riences, représentations, descriptions, images ou formalisations mathématiques. Dans cette zone, il n'y a aucun niveau de Réalité.

La non-résistance de cette zone de transparence absolue est due, tout simplement, aux limitations de notre corps et de nos organes des sens, quels que soient les instruments de mesure qui prolongent ces organes des sens. La zone de non-résistance correspond à ce qui ne se soumet à aucune rationalisation. Il convient de se rappeler la distinction importante faite par Edgar Morin entre rationnel et rationalisation ${ }^{8}$. La zone de nonrésistance est rationnelle, mais elle n'est pas rationalisable. Elle traduit l'existence d'une interaction entre le Sujet et l'Objet, irréductible au Sujet ou à l'Objet. Cette interaction est tout d'abord une expérience, qui se traduit par un sentiment de ce qui relie les êtres et les choses et, par conséquent, qui induit dans les tréfonds de l'être humain le respect absolu des altérités unies par la vie commune sur une seule et même Terre.

La structure ouverte de l'ensemble des niveaux de Réalité est en accord avec un des résultats scientifiques les plus importants du XXe siècle : le théorème de Gödel, concernant l'arithmétique. Le théorème de Gödel nous dit qu'un système d'axiomes suffisamment riche conduit inévitablement à des résultats soit indécidables, soit contradictoires. La portée du théorème de Gödel a une importance considérable pour toute théorie moderne de la connaissance car il ne concerne pas que le seul domaine de l'arithmétique, mais aussi toute mathématique qui inclut l'arithmétique. La structure gödelienne de l'ensemble des niveaux de Réalité, associée à la logique du tiers inclus, implique l'impossibilité de bâtir une théorie complète pour décrire le passage d'un niveau à l'autre et, a fortiori, pour décrire l'ensemble des niveaux de Réalité. L'unité reliant tous les niveaux de Réalité, si elle existe, doit nécessairement être une unité ouverte.

Un nouveau Principe de relativité émerge de la coexistence entre la pluralité complexe et l'unité ouverte : aucun niveau de

8 Edgar Morin, "Anthropologie de la connaissance ", dans La méthode, tome III, La connaissance de la connaissance, Seuil, Paris, 1986. 
Réalité ne constitue un lieu privilégié d'où l'on puisse comprendre tous les autres niveaux de Réalité. Un niveau de Réalité est ce qu'il est parce que tous les autres niveaux existent à la fois. Ce Principe de relativité est fondateur d'un nouveau regard sur la culture, la religion, la politique, l'art, l'éducation, la vie sociale. Et lorsque notre regard sur le monde change, le monde change. "Dire un mot vrai équivaut à la transformation du monde "écrit le grand pédagogue brésilien Paulo Freire dans Pédagogie des opprimés.

Selon la vision transdisciplinaire, la connaissance n'est ni extérieure, ni intérieure : elle est à la fois extérieure et intérieure. L'étude de l'Univers et l'étude de l'être humain se soutiennent l'une l'autre. Les sciences exactes et les sciences humaines sont complémentaires. C'est la transdisciplinarité qui est le tiers permettant leur interaction.

On peut conclure que les sciences exactes, les sciences humaines et la transdisciplinarité constituent le ternaire de la connaissance, permettant l'émergence d'une nouvelle forme d'humanisme qui offre à chaque être humain la capacité maximale de développement culturel et spirituel. Il s'agit de chercher ce qu'il y a entre, à travers et au delà des êtres humains - ce qu'on peut appeler l'Être des êtres. 


\section{Bibliographie}

Heisenberg, Werner, Philosophie - Le manuscrit de 1942, trad. de l'allemand et introduction par Catherine Chevalley, Paris, Seuil, 1998 [éd. allemande: 1984].

Lupasco, Stéphane, Le principe d'antagonisme et la logique de l'énergie Prolégomènes à une science de la contradiction, Monaco, Le Rocher, coll. «L'esprit et la matière », [1951] 1987.

Morin, Edgar, "Anthropologie de la connaissance ", dans La méthode, tome III, La connaissance de la connaissance, Seuil, Paris, 1986.

Nicolescu, Basarab, La transdisciplinarité, manifeste, Rocher, Monaco, 1996.

Nicolescu, Basarab, Nous, la particule et le monde, Rocher, Monaco, 2002.

Nicolescu, Basarab, Qu'est-ce que la Réalité?, Liber, Montréal, 2009.

Piaget, Jean, "L'épistémologie des relations interdisciplinaires " dans L'interdisciplinarité; Problèmes d'enseignement et de recherche dans les universités, OCDE, Paris, 1972, p. 131-144.

Snow, Charles Percy, The Two Cultures, Cambridge University Press, Cambridge, 1993. 